\title{
ANÁLISE MULTIVARIAdA DE FATORES DE RISCO PARA O BAIXO PESO AO NASCER EM NASCIDOS VIVOS DO MUNICIPIO DE SÃO PAULO, SP (BRASIL)
}

\author{
Maria Helena D'Aquino Benicio* \\ Carlos Augusto Monteiro* \\ José Maria Pacheco de Souza** \\ Euclides Aires de Castilho*** \\ Isildinha Marques dos Reis Lamonica*
}

\begin{abstract}
BENICIO, M.H.D'A. et al. Análise multivariada de fatores de risco para o baixo peso ao nascer em nascidos vivos do município de São Paulo, SP (Brasil). Rev. Saúde públ., S. Paulo, 19:311-20, 1985.
\end{abstract}

RESUMO: Em casuística de 20.850 nascidos vivos não gemelares ocorridos em 31 maternidades do Município de São Paulo, SP, Brasil (parte da casuística total do Estudo Antropométrico do Recém-nascido Brasileiro), procurou-se identificar fatores de risco para o baixo peso ao nascer (peso $\leqslant 2.500 \mathrm{~g}$ ), tendo sido estipulados a priori, para estudo, os fatores escolaridade materna, estado marital, idade materna, paridade, peso pré-gestacional, tabagismo na gravidez e assistência pré-natal. A partir de análise multivariada pela técnica de modelos log-lineares, foram identificados quatro fatores de risco significativos: "ausência de assistência pré-natal", "peso prégestacional $<50 \mathrm{~kg}$ ", "tabagismo na gestação" e "idade materna $<20$ anos". O risco relativo associado a "ausência de pré-natal" foi de 2,2 nas mães de escolaridade inferior ao ginásio completo e de 3,4 nas de escolaridade igual ou superior àquele nível. Os riscos associados às características maternas de peso, tabagismo e idade foram respectivamente $1,9,1,7$ e 1,4 e independentes da escolaridade materna. Considerando-se além da magnitude dos riscos detectados a frequiência com que os fatores de risco se apresentaram na população, constatou-se que o peso pré-gestacional insuficiente, o tabagismo na gestação e a ausência de assistência pré-natal, particularmente em mães de "baixa escolaridade", são fatores cujo controle exerceria importante decréscimo na incidência de recém-nascidos de baixo peso. Assim sendo, tais fatores deveriam ser cuidadosamente considerados em programas de intervenção.

UNITERMOS: Baixo peso ao nascer. Análise multivariada. Idade materna. Peso pré-gestacional. Tabagismo. Assistência pré-natal.

\section{INTRODUÇÃO}

A condição de baixo peso ao nascer (peso de nascimento $\leqslant 2.500 \mathrm{~g}$ ) associam-se inúmeras desvantagens biológicas para o concepto, envolvendo alterações respiratórias e metabólicas de grave repercussão no pós-parto imediato, diminuição da competência imunológica e prejuízo ao crescimento e desenvolvimento pós-natais ${ }^{5,11,12,14,26}$. Estudos realizados em países de diferentes níveis de desenvolvimento têm demonstrado, por sua vez, ser a condição de peso ao nascer um dos principais fatores, senão o principal, a determinar a probabilidade de

\footnotetext{
- Do Departamento de Nutrição da Faculdade de Saúde Pública da Universidade de São Paulo Av. Dr. Armaldo, 715 - 01255 - São Paulo. SP - Brasil.

* Do Departamento de Epidemiologia da Faculdade de Saúde Pública da Universidade de São Paulo.

** Do Departamento de Medicina Preventiva da Faculdade de Medicina da Universidade de São Paulo - Av. Dr. Arnaldo, 455 - 01246 - São Paulo, SP — Brasil.
} 
BENíCIO, M.H.D'A. et al. Análise multivariada de fatores de risco para o baixo peso ao nascer em nascidos vivos do município de São Paulo, SP (Brasil). Rev. Saúde públ., S. Paulo, 19:311-20, 1985 .

um recém-nascido sobreviver ao período neo-natal e mesmo a todo o restante primeiro ano de vida ${ }^{6,10,17,18}$.

A incidência de recém-nascidos de baixo peso é relativamente bem conhecida em países desenvolvidos, onde abrange 5 a $8 \%$ dos nascidos-vivos ${ }^{15}$. E menos conhecida nos países não desenvolvidos, para os quais se dispõe de estudos de modo geral realizados apenas em determinadas frações da população, onde podem ser verificadas incidências de 13 a $43 \%{ }^{15}$. A Organização Mundial de Saúde estima em, respectivamente, $7 \%$ e $18 \%$ as incidências médias que caracterizam os países desenvolvidos e em desenvolvimento ${ }^{27}$.

A determinação da condição de baixo peso ao nascer é processo reconhecidamente complexo que atende a modelo multicausal e envolve diferentes fatores de risco. Em países desenvolvidos, estudos realizados em casuísticas apropriadas e utilizando adequadas técnicas multivariadas de análise têm apontado diversas condições maternas - relacionadas a aspectos sócio-econômicos, história reprodutiva, características antropométricas, hábito de fumar e assistência pré-natal - como fatores que elevariam significativamente a probabilidade de baixo peso ao nascer ${ }^{3,4,7,9,13,20,22,24,25}$.

Em nosso meio, bem como de modo geral nos demais países não desenvolvidos, dispõe-se de escassos estudos sobre fatores de risco de baixo peso ao nascer $e$, ainda assim, frequientemente realizados em casuísticas não suficientemente grandes que permitam a investigação simultânea de fatores e a aplicação de adequadas técnicas multivariadas de análise.

O objetivo do presente estudo é o de identificar, por meio de análise multivariada, fatores de risco significativamente associados ao baixo peso ao nascer em uma casuística de mais de 20.000 nascidos vivos, ocorridos em 1978, em 31 maternidades do município de São Paulo, privilegiando-se o exame de variáveis cuja associação com peso ao nascer já tenha sido documentada na literatura e que possam ensejar ações de prevenção e/ou controle a nível de serviços de assistência à saúde.

\section{MATERIAL E METODOS}

A presente investigação foi realizada a partir da casuística total de 30.834 mães e respectivos recém-nascidos vivos não gemelares incluídos no "Estudo Antropométrico do Recém-Nascido Brasileiro" realizado no município de São Paulo em 1978*. Tais nascimentos foram provenientes de partos ocorridos em 31 hospitais-maternidades que participaram do referido estudo e não constituem, nesta medida, uma amostra probabilística de nascidos vivos do município. Em publicação anterior ${ }^{1}$ pôde-se demonstrar, entretanto, que o caráter voluntário da participação dos hospitais não originou maiores peculiaridades na casuística estudada. Em relação a três importantes variáveis maternas - idade, altura e peso - a casuística apresentou distribuição praticamente superposta àquela esperada para o município como um todo. Para esta investigação, foram considerados apenas os recém-nascidos que apresentaram informações completas para todas as variáveis pesquisadas, ou seja, 20.850 recém-nascidos ou $67 \%$ da casuística total. $\mathrm{Na}$ mesma publicação já mencionada ${ }^{1}$, demonstrou-se que o conjunto de recém-nascidos não incluídos na presente investigação acusou frequiencias de fatores de risco e incidência de baixo peso ao nascer semelhantes às observadas para a casuística efetivamente investigada.

\footnotetext{
* Estudo coordenado pelo Prof. Fernando José de Nóbrega com o apoio da Sociedade Brasileira de Pediatria. (Em publicação no Jornal de Pediatria.)
} 
BENICIO, M.H.D'A. et al. Análise multivariada de fatores de risco para o baixo peso ao nascer em nascidos vivos do município de São Paulo, SP (Brasil). Rev. Saúde públ., S. Paulo, 19:311-20, 1985.

A coleta de dados do "Estudo Antropométrico do Recém-Nascido Brasileiro" foi feita de modo padronizado a partir de entrevistas a puérperas complementadas por informaçōes transcritas de seus prontuários, envolvendo a participação de médicos-berçaristas e de outros funcionários especialmente designados pela direção de cada berçário.

Além da condição de peso ao nascer, a presente investigação incluiu o estudo das seguintes variáveis maternas: escolaridade, estado marital, idade, paridade, peso pré-gestacional, tabagismo e assistência pré-natal (esta última definida como o recebimento de pelo menos três consultas). A partir destas variáveis foram estipulados os seguintes fatores de risco para o baixo peso ao nascer: "escolaridade inferior ao primeiro grau completo" (que será indicado doravante como "baixa escolaridade"), "mães sem companheiro", "idade $<20$ anos", "primiparidade", "peso < $50 \mathrm{~kg}$ ", "tabagismo durante a gestação" e "ausência de assistência pré-natal". A orientação utilizada para se estabelecer níveis de corte para as variáveis dicotômicas (escolaridade, idade, paridade e peso) baseou-se em informações da literatura e na observação da distribuição não ajustada da variável peso ao nascer segundo aquelas variáveis maternas.

O efeito próprio de cada fator de risco sobre a incidência de recém-nascidos de baixo peso foi estudado por meio de análise multivariada conduzida pela técnica de modelos log-lineares ${ }^{2,23}$.

A técnica de modelos log-lineares consiste, sucintamente, em se procurar ajustar uma função linear ao logarítimo das freqüências de cada casela de uma tabela de contingência obtida a partir do cruzamento simultâneo de variáveis. No caso presente em que o interesse é verificar efeitos independentes ("fatores de risco") sobre uma dada variável resposta ("peso ao nascer"), adotou-se a especificação logito da técnica de modelos log-lineares. Nesta situação, procurou-se ajustar um modelo que, contendo todos os parâmetros referentes às associações entre variáveis independentes, contenha o menor número possível de parâmetros referentes a associações que incluam a variável resposta. Para se chegar a este menor número possível, são descartados todos aqueles parâmetros que não contribuem significativamente para o ajuste do modelo, ou, mais especificamente, todos aqueles parâmetros cuja retirada possa ser feita sem que o $\chi^{2}$ do teste da razão de máxima verossimilhança $\left(\chi^{2}\right.$ RMv $)$ do modelo sofra decréscimo significativo (para $\alpha$ de $5 \%$ e 1 g.l. aquele decréscimo não pode ultrapassar 3,84). Ao esgotarem-se todas as retiradas possiveis, chega-se ao modelo final, cujo ajuste às frequiências observadas na tabela de contingência inicial é conhecido por meio de exame do valor do $\chi^{2}$ RMv relativo aos graus de liberdade correspondentes ao total de parâmetros retirados.

A partir do modelo obtido pela análise multivariada dos dados, foram estabelecidas as frequiências esperadas da variável peso ao nascer na ausência e na presença de fatores de risco considerados pelo modelo, o que possibilitou o cálculo de razões de produtos cruzados ("odds ratio"), entendidas aquelas como satisfatórias estimativas dos riscos relativos associados a presença individual ou simultânea daqueles fatores ${ }^{8}$.

\section{RESULTADOS}

\section{Modelo obtido pela análise multivariada}

A análise multivariada conduzida pela técnica de modelos log-lineares levou a um modelo simplificado final em que permaneceram apenas 7 das 127 possíveis associações entre fatores de risco 
BENICIO, M.H.D'A. et al. Análise multivariada de fatores de risco para o baixo peso ao nascer em nascidos vivos do município de São Paulo, SP (Brasil). Rev. Saúde públ., S. Paulo, 19:311-20, 1985 .

e combinações de fatores de risco e peso ao nascer. Tal modelo, apesar de privado dos parâmetros referentes às 120 associações descartadas, apresentou um bom ajuste médio em relação às frequiências observadas a partir do cruzamento simultâneo de todas as variáveis estudadas: $\chi^{2}$ RMv de 118,73 com 120 graus de liberdade $(p=0,52)$.

As estimativas dos parâmetros referentes às sete associações entre fatores de risco e peso ao nascer que contribuíram significativamente para o ajuste do modelo são apresentadas na Tabela 1. A partir dali, verificou-se que o modelo escolhido descartou a associação "simples" entre primiparidade e peso ao nascer bem como todas as associações entre interações de fatores de risco e peso ao nascer, com a única exceção da interação "ausência de pré-natal-baixa escolaridade".

TABELA 1

Estimativas padronizadas dos parâmetros do modelo escolhido referentes às associaçöes entre fatores de risco e baixo peso ao nascer.

\begin{tabular}{lcc}
\hline $\begin{array}{l}\text { Fatores de risco } \\
\text { associados ao baixo } \\
\text { peso ao nascer }\end{array}$ & $\begin{array}{c}\text { Estimativa } \\
\text { padronizada } \\
\text { do parâmetro* }\end{array}$ & $\begin{array}{c}\text { Significado } \\
\text { estatístico }\end{array}$ \\
"Baixa escolaridade" & 0,480 & 0,63 \\
"Mães sem companheiro" & 1,508 & 0,13 \\
"Idade < 20 anos" & 1,974 & $<0,05$ \\
"Peso < 50 kg" & 3,876 & $<0,001$ \\
"Tabagismo" & 3,001 & $<0,01$ \\
"Ausência de pré-natal" & 5,051 & $<0,0001$ \\
"Ausência de pré-natal-baixa escolaridade" & 2,094 & $<0,05$ \\
\hline
\end{tabular}

* Obtida pela divisão do parâmetro pelo seu respectivo desvio-padrão, tendo distribuição normal reduzida.

E importante assinalar que o ajuste obtido pelo modelo escolhido não diferiu do ajuste obtido por modelos imediatamente mais complexos em que estavam presentes os parâmetros referentes às associações descartadas. Por outro lado, tentativas de simplificações adicionais do modelo escolhido resultaram sempre em decréscimo significativo do ajuste, não sendo, portanto, efetivadas.

Uma última observação que deve ainda ser feita em relação ao modelo escolhido é que dois dos sete parâmetros que aparecem na Tabela 1 não chegam a alcançar significado estatístico a nível de $\alpha=5 \%$, não caracterizando, nesta medida, associações significativas com o peso ao nascer. No caso do parâmetro relativo a "mães sem companheiro", a permanência do mesmo no modelo se deve, efetivamente, à diminuição significativa de ajuste provocada pela sua retirada. No caso do parâmetro relativo a "baixa escolaridade", a permanência do mesmo se deve ao decréscimo de ajuste que a retirada do parâmetro referente à interação "ausência de pré-natal-baixa escolaridade" provocaria (o modelo sendo hierárquico exige a associação "simples" quando outra mais "complexa" está presente).

\section{Estimativa de riscos relativos}

Em sua primeira linha, a Tabela 2 apresenta a distribuição da variável peso 
BENICIO, M.H.D'A. et al. Análise multivariada de fatores de risco para o baixo peso ao nascer em nascidos vivos do município de São Paulo, SP (Brasil). Rev. Saúde públ., S. Paulo, 19:311-20, 1985.

ao nascer (baixo peso-não baixo peso) ajustada pelo modelo para a situação em que nenhum dos fatores de risco considerados estivesse presente. Nas seis linhas subseqüientes da mesma Tabela, são apresentadas as distribuições ajustadas para a presença individual e exclusiva de cada um dos fatores de risco considerados pelo modelo, bem como a estimativa do risco relativo de baixo peso fornecida pela razão de produtos cruzados ("odds ratio"). Evidencia-se que as estimativas

TABELA 2

Frequiências ajustadas de peso ao nascer e razão dos produtos cruzados ("odds ratio") conforme a presença de determinados fatores de risco.

\begin{tabular}{|c|c|c|c|c|c|}
\hline \multirow{2}{*}{ Fatores de risco presentes } & \multicolumn{2}{|c|}{$\begin{array}{l}\text { Frequiências ajustadas de } \\
\text { peso ao nascer }\end{array}$} & \multirow{2}{*}{\multicolumn{3}{|c|}{ Razão dos produzos cruzados* }} \\
\hline & baixo peso & não baixo peso & & & \\
\hline Nenhum & 50,70 & 1543,30 & & & \\
\hline "Baixa escolaridade" & 158,60 & 3657,40 & 1,320 & $(0,853$ & - 2,035) \\
\hline "Mães sem companheiro" & 0,90 & 21,10 & 1,298 & $(0,926$ & $-1,817)$ \\
\hline "Idade $<20$ anos" & 1,81 & 39,19 & 1,406 & $(1,003$ & - 1,967$)$ \\
\hline "Peso < $50 \mathrm{~kg} "$ & 25,07 & 391,93 & 1,947 & $(1,393$ & - 2,732$)$ \\
\hline "Tabagismo" & 37,35 & 678,65 & 1,675 & $(1,196$ & - 2,347$)$ \\
\hline "Ausência de pré-natal" & 3,43 & 30,57 & 3,415 & $(1,816$ & - 6,420$)$ \\
\hline $\begin{array}{l}\text { "Ausência de pré-natal- } \\
\text { baixa escolaridade" }\end{array}$ & 31,54 & 437,46 & 2,195 & $(1,390$ & 3,451 ) \\
\hline
\end{tabular}

* Sendo $a$ e $b$ as freqüências ajustadas de baixo peso e não baixo peso na ausência de fatores de risco e $c$ e $d$ as mesmas freqüências ajustadas para a presença de um determinado fator; a razão dos produtos cruzados referentes àquele fator é dada por b.c/a.d .

mais elevadas de risco são encontradas para "ausência de pré-natal", 3,4 , e para "peso < $50 \mathrm{~kg}$ ", 1,9 .

Estimativas menores, porém ainda significativas, são encontradas para "tabagismo", 1,7 , e para "idade $<20$ anos", 1,4 . As estimativas de risco para os dois restantes fatores, "baixa escolaridade" e "mães sem companheiro", estiveram em torno de 1,3 e não chegaram a alcançar significado estatístico.

Ainda na Tabela 2, é apresentada a distribuição da variável peso ao nascer ajustada pelo modelo para a presença exclusiva da interação "ausência de prénatal-baixa escolaridade", verificando-se que nesta situação a estimativa de risco de baixo peso é de 2,2 .
$\mathrm{Na}$ medida em que o modelo é multiplicativo $^{2}$ e não cogitou de outras interações além da "ausência de pré-natalbaixa escolaridade", as estimativas de risco para todas as demais combinações de fatores de risco podem ser conhecidas pela simples multiplicação das razões de produtos cruzados referentes à presença individual de cada fator. Assim, por exemplo, se estivessem presentes simultaneamente "tabagismo", "peso < $50 \mathrm{~kg}$ " e "idade $<20$ anos", o risco estimado de baixo peso seria de $1,947 \times 1,675 \times$ $1,406=4,585$. Nesta situação específica, a incidência mínima de recém-nascidos de baixo peso de $3,18 \%$ (incidência obtida a partir das frequências ajustadas pelo modelo para a ausência completa de fatores de risco) tenderia a se aproximar de $15 \%$. 
BENICIO, M.H.D'A. et al. Análise multivariada de fatores de risco para o baixo peso ao nascer em nascidos vivos do município de São Paulo, SP (Brasil). Rev. Saúde públ., S. Paulo, 19:311-20, 1985.

\section{DISCUSSÃO}

Em nascidos-vivos não gemelares do município de São Paulo, procurou-se identificar fatores de risco para baixo peso ao nascer e quantificar riscos relativos associados à presença dos mesmos, tendo sido estipulados a priori para estudo os fatores "baixa escolaridade materna", "mães sem companheiro", "idade $<20$ anos", "peso prévio à gravidez $<50 \mathrm{~kg}$ ", "tabagismo na gestação" e "ausência de assistência pré-natal".

Por meio de análise multivariada apropriada para identificar os efeitos próprios de cada fator sobre o peso ao nascer, observou-se que três deles não chegaram a exercer influência significativa sobre a incidência de baixo peso: "baixa escolaridade", "mães sem companheiro" e "primiparidade". Os restantes quatro fatores, em diferentes graus, elevaram significativamente a incidência daquela condição: "idade < 20 anos", 1,4 vezes, "peso < $50 \mathrm{~kg}$ ", 1,9 vezes, "tabagismo", 1,7 vezes e "ausência de pré-natal", 2,2 vezes nas mães de "baixa escolaridade" e 3,4 vezes nas mães de "alta escolaridade".

A influência não significativa da variável escolaridade, entendida esta como uma aproximação do estrato social materno, deve ser interpretada com cautela, uma vez que na análise foram incluídas outras variáveis independentes, como assistência pré-natal e peso pré-gestacional, que, teoricamente, podem representar importantes vias de expressão da determinação sócio-econômica de peso ao nascer. Em relação a este aspecto, cumpre mencionar que todos os quatro fatores de risco significativamente associados ao baixo peso mostram associação de mesmo sentido com a "baixa escolaridade", destacando-se em particular os fatores "ausência de pré-natal" e "idade $<20$ anos", cujas freqüências na "baixa escolaridade" excederam, respectivamente, em cerca de quatro vezes e duas vezes as freqüências encontradas na "alta escolaridade" $(13,7 \% \times 3,1 \%$, no primeiro caso, e $13,1 \% \times 7,5 \%$, no segundo caso). Também é importante notar que, na ausência do controle de outras variáveis, foi efetivamente significativa a associação entre "baixa escolaridade" e baixo peso ao nascer ( $p<0,001)$ atribuindose, então, àquela condição risco relativo próximo a 1,6.

A influência não significativa da outra variável que, de certa forma, também expressa condições sócio-econômicas maternas - o estado marital - deve igualmente ser interpretada com cautela, pela mesma razão já aventada no caso da escolaridade. Para estado marital, também observou-se que todos os fatores de risco significativos foram mais frequientes na categoria sócio-econômica desfavorável, destacando-se, desta vez, os fatores "ausência de pré-natal" e "tabagismo" cujas freqüências em "mães sem companheiro" excederam, respectivamente, em cerca de três vezes e uma e meia vezes as frequiências encontradas em "mães com companheiros" $(25,7 \%$ x $8,9 \%$, no primeiro caso, e $50,5 \% \times 34,3 \%$, no segundo caso). Por outro lado, o exame da relação estado marital-peso ao nascer, na ausência do controle de outras variáveis, indicou ser a condição "mães sem companheiro" fator significativamente associado a baixo peso ao nascer ( $\mathrm{p}<0,001)$, atribuindo àquela condição risco relativo em torno de 1,5.

Assim, tanto no caso da escolaridade materna quanto no caso do estado marital, as observações feitas sugerem que os achados da análise multivariada não devam ser interpretados como demonstrativos da não-importância da influência daquelas variáveis sobre o peso ao nascer e, menos ainda, como demonstrativos da não relevância da determinação sócio-econômica da condição de baixo peso. Os referidos achados apenas indicam não existir efeitos significativos das 
BENíCIO, M.H.D'A. et al. Análise multivariada de fatores de risco para o baixo peso ao nascer em nascidos vivos do município de São Paulo, SP (Brasil). Rev. Saúde públ.. S. Paulo, 19:311-20, 1985

variáveis escolaridade e estado marital que sejam independentes das demais variáveis estudadas. Igualmente não seria válido concluir que a influência sócioeconômica sobre o peso ao nascer se dê exclusivamente através de assistência prénatal, tabagismo e idade e peso maternos, uma vez que as variáveis escolaridade e estado marital são simples aproximações disponíveis do estrato social da mãe e limitados indicadores do conjunto de condições ambientais capazes de influenciar o peso ao nascer.

A revisão de outros estudos de análise multivariada, que tenham procurado identificar na escolaridade e no estado marital da mãe fatores de risco para o baixo peso ao nascer, não aponta resultados em uma única direção. Os estudos de Quick e col. ${ }^{2: 2}$ e de Wiener e Milton ${ }^{25}$, por exemplo, não identificam naquelas condições fatores de risco para o baixo peso ao nascer. Já os estudos de Bross e Shapiro ${ }^{3}$ e de Eisner e col. ${ }^{9}$ apontam em direção oposta. 0 diferente significado que variáveis como escolaridade e estado marital podem assumir em diferentes contextos sociais, somado às inevitáveis diferenças metodológicas quanto à definição de categorias de risco, de variáveis sob controle e de técnicas de análise, tornam demasiadamente complexas as comparações dos resultados obtidos por aqueles estudos com os nossos resultados.

A influência não significativa da variável paridade merece interpretação diversa da efetuada para escolaridade e estado marital, pois, neste caso, as demais variáveis independentes incluídas na análise não podem ser entendidas como vias de expressão dos efeitos da variável sobre o peso ao nascer. Observando-se a relação "primiparidade"-baixo peso ao nascer, na ausência de controle de outras variáveis, verifica-se associação estatística entre as duas condições $(p<0,005)$, porém de fraca intensidade (risco relativo inferior a 1,2). Tal associação não subsiste na análise multivariada, basicamente em decorrência, neste caso, do controle da idade materna, variável da qual a paridade depende diretamente $(20,5 \%$ das mães primíparas têm menos de 20 anos, enquanto apenas $5,7 \%$ das multíparas estão naquela faixa etária). Nesta medida pode-se efetivamente identificar como espúria a associação aparente entre "primiparidade" e baixo peso ao nascer.

A identificação na casuística estudada dos fatores "idade jovem", "peso prégestacional insuficiente", "tabagismo na gestação" e "inadequada assistência prénatal" como significativos fatores de risco para o baixo peso ao nascer confirma os achados de diversos estudos de análise multivariada realizados em países desenvolvidos e já referidos na introdução deste artigo $3,4,7,9,13,20,22,24,25$

O relativo alto risco de "inadequada assistência pré-natal", identificado no presente estudo, já havia sido evidenciado por Eisner e col." que encontraram na população americana riscos de magnitude variando entre 2,0 e 4,0. A mesma investigação identificou riscos menores para o fator "idade jovem", variando entre 1,3 e 1,6 e, portanto, próximos ao risco por nós encontrado.

Um grande número de estudos tem abordado o papel do tabagismo no aumento do risco de baixo peso ao nascer. Meyer ${ }^{14}$, revisando tais estudos, constatou que a presença do tabagismo usualmente duplica a incidência de recémnascidos de baixo peso - fato próximo ao encontrado por nossa investigação.

Não encontramos na literatura estimativas ajustadas de risco de baixo peso ao nascer para "peso pré-gestacional insuficiente" que permitissem comparações com nossos achados. Em estudo posterior $^{21}$ que realizamos em oito materni- 
BENICIO, M.H.D'A. et al. Análise multivariada de fatores de risco para o baixo peso ao nascer em nascidos vivos do município de São Paulo, SP (Brasil). Rev. Saúde públ., S. Paulo, 19:311-20, 1985.

dades do município de São Paulo e que atendem especialmente gestantes de baixo nível sócio-econômico, por meio da mesma técnica de análise multivariada de modelos log-lineares, identificamos, para peso pré-gestacional inferior a $45 \mathrm{~kg}$, risco de 1,86 .

Do ponto de vista da saúde pública, o conhecimento do risco relativo que um dado fator determina para uma condição mórbida é importante elemento para a orientação de suas ações. Porém, ainda de melhor valia, neste caso, é o conhecimento do risco atribuivel do referido fator na população, ou seja, a proporção de casos da doença que poderia ser atribuída, na população em questão, à presença do fator de risco, ou, alternativamente, o decréscimo proporcional na incidência da doença que poder-se-ia esperar se a população não mais estivesse exposta ao fator ${ }^{16}$. Tal índice, evidentemente, será tanto maior quanto maior o risco relativo determinado pelo fator e quanto maior a frequiência do fator na população. E calculado, de forma bastante simples, por $\frac{b(r-1)}{b(r-1)+1}$, onde $b$ é a frequêencia do fator de risco na população e $r$ seu risco relativo ${ }^{16}$.

Observando a Tabela 3 têm-se os fatores de risco para o baixo peso ao nascer identificados na casuística estudada segundo o risco atribuível associado a cada um deles.

TABELA 3

Fatores de risco de baixo peso ao nascer

\begin{tabular}{lccc}
\hline & $\begin{array}{c}\text { Freqüência do } \\
\text { fator na } \\
\text { população \% }\end{array}$ & Risco relativo* & Risco Atribuível \\
\hline "Peso < $50 \mathrm{~kg} "$ & $\mathbf{2 8 , 9 \%}$ & 1,95 & $\mathbf{2 1 , 0 \%}$ \\
"Tabagismo" & $\mathbf{3 7 , 8 \%}$ & 1,67 & $\mathbf{2 0 , 0 \%}$ \\
"Ausência de pré-natal- & $9,5 \%$ & 2,19 & $\mathbf{1 0 , 0 \%}$ \\
$\begin{array}{l}\text { baixa escolaridade" } \\
\text { "Idade < 20 anos" }\end{array}$ & $11,4 \%$ & 1,40 & $4,0 \%$ \\
"Ausência de pré-natal- & $0,9 \%$ & $\mathbf{3 , 4 1}$ & $\mathbf{2 , 0 \%}$ \\
alta escolaridade" & & & \\
\hline
\end{tabular}

* Estimado a partir da razão dos produtos cruzados.

Pode-se notar o papel preponderante que o "peso pré-gestacional insuficiente" e o "tabagismo", e em menor escala também a ausência de assistência pré-natal, assumem na determinação do baixo peso ao nascer.

Estes últimos resultados recomendam - aprofundamento da análise sobre a viabilidade e as perspectivas do controle em nosso meio dos três fatores de risco mencionados e sobre as medidas mais oportunas que deveriam ser tomadas ao nível da política e dos programas de saúde pública. Desde logo, entretanto, é possível antever que uma estratégia de expansão e melhoria de qualidade dos programas de assistência pré-natal poderia ser uma bem sucedida resposta de curto prazo. Tal estratégia deveria permitir cobrir a relativa pequena proporção de gestantes ainda não atendidas por programas de assistência pré-natal no município, sobretudo aquelas de pior nível sócio-econômico, onde a cobertura da assistência é mais precária. Simultaneamente deveria dotar a assistência prénatal de conteúdo programático apropriado para fazer frente ao controle dos 
BENfCIO, M.H.D'A. et al. Análise multivariada de fatores de risco para o baixo peso ao nascer em nascidos vivos do município de São Paulo, SP (Brasil). Rev. Saúde públ., S. Paulo, 19:311-20, 1985

problemas mais relevantes na determinação imediata do baixo peso ao nascer, o que deveria incluir, evidentemente, a detecção e o desestímulo ao tabagismo na gestação e a correção de eventuais déficits nutricionais presentes ao início ou durante a gestação.

\section{AGRADECIMENTO}

Ao Prof. Fernando José de Nóbrega, por ter cedido os dados originais do "Estudo Antropométrico do Recém-nasciđo Brasileiro", para realização do presente trabalho.

BENICIO, M.H.D'A. et al. [Multivariate analysis of risks factors for low birthweight in livebirths in the city of S. Paulo, SP (Brazil)]. Rev. Saúde públ., S. Paulo, 19:311-20, 1985.

ABSTRACT: An investigation, as part of a national study on anthropometric characteristics of livebirths, was carried out on 20,850 single livebirths born in 31 maternity hospitals in S. Paulo, Brazil, into risk factors for low birth weight (weight less than 2,501 gr.). The following factors were studied: maternal schooling, marital status, maternal age, parity, prepregnancy weight, smoking during pregnancy and prenatal care. Through multivariate analyses using log linear models, four significative risk factors for low birth weight were identified: "no prenatal care", prepregnancy weight $<50 \mathrm{~kg}$ ", "smoking during pregnancy" and "maternal age $<20$ years". The relative risk for "no prenatal care" was 3.4 in mothers with 8 or more years of schooling and 2.2 in mothers with less schooling. The risks associated with prepregnancy weight, smoking and maternal age were, respectively, 1.9, 1.7 and 1.4 and were found to be independent of maternal schooling. Considering simultaneously risks, magnitude and frequency of the risk factors in the population, it is evident that prepregnancy weight, smoking during pregnancy and prenatal care particularly for mothers with low socio-economic level, are factors whose control would result in considerable decrease in low birth weight incidence and, for that reason. they should be carefully considered by health intervention programs.

UNITERMS: Low birth weight. Multivariate analysis. Maternal age. Prepregnancy weight. Smoking. Prenatal care.

\section{REFERENCIAS BIBLIOGRÄFICAS}

1. BENiCIO, M.H.D'A. Fatores de risco de baixo peso ao nascer em recém-nascidos vivos, Município de São Paulo, 1978. São Paulo, 1983. [Tese de Doutoramento - Faculdade de Medicina da USP].

2. BISHOP, Y.M.M. et al. Discrete multivariate analysis: theory and practice. Cambridge, Mass., The MIT Press, 1980.

3. BROSS, D.S. \& SHAPIRO, S. Direct and indirect associations of five factors with infant mortality. Amer. J. Epidem., 115: 78-91, 1982.

4. BUTLER, N.R. et al. Cigarrette smoking in pregnancy: its influence on birth weight and perinatal mortality. Brit. med. J., 2:127-30, 1972.

5. CHANDRA, R.K. Fetal mal nutrition and post natal immuno competence. Amer. J. Dis. Child., 129:450-4, 1975.
6. CHASE, H.C. International comparison of perinatal and infant mortality: the United States and six West European Countries. Vital Hlth Stat. Ser. 3, (6) Mar. 1967.

7. COMSTOCK, G.W. et al. Low birth weight and neonatal mortality rate related to maternal smoking and socioeconomic status. Amer. J. Obstet. Gynec., 111:53-9, 1971.

8. CORNFIELD, J. A method for estimating comparative rates from clinical data: application to cancer of lung, breast and cervix. J. Nat. Cancer Inst., 11:1269. 75,1951

9. EISNER, V. et al. The risk of low birth weight. Amer, J. publ. Hlth, 69:887-93, 1979. 
BENICIO, M.H.D'A, et al. Análise multivariada de fatores de risco para o baixo peso ao nascer em nascidos vivos do município de São Paulo, SP (Brasil). Rev. Salíde públ., S. Paulo, 19:311-20, 1985.

10. ERHARDT, C.L. et al. Influence of weight and gestational age on perinatal and neonatal mortality by ethinic group. Amer. J. publ. Hlth, 54:1841-55, 1964.

11. FITZHARDINGE, P.M. \& STEVEN, E.M. The small-for-date infant. II Neurological and intellectual sequelae. Pediatrics, 50:50-7, 1972.

12. HARPER, P.A. \& WIENER, G. Sequelae of low birth-weight. Ann. Rev. Med. 16:405-20, 1965.

13. KULLANDER, S. \& KALLEN, B. A prospective study of smoking and preg. nancy. Acta obstet.gynec.scand., 50:83. 94, 1971.

14. LASKI, R.E. et al. Birth-weight and psychomotor performance in rural Guatemala. Amer. J. Dis. Child., 129:566-70, 1975.

15. LECHTIG, A. et al. Maternal nutrition and fetal growth in developing societies: socioeconomic factors. Amer. J. Dis. Child., 129:434-7, 1975.

16. LILIENFELD, A.M. Retrospective and cross sectional studies. In: Lilienfeld, A.M. Foundation of epidemiology. New York, Oxford University Press, 1976 p. 191-225.

17. MATA, L. The children of Santa Maria Cauqué: a prospective study of health and growth. Cambridge, Mass., The MIT Press, 1977.

18. MCKEOWN, T. \& GIBSON, J.R. Observations on all births $(23,790)$ in Birminghan, 1947. IV - "Premature birth". Brit. med. J., 3:513-7, 1951.

19. MEYER, M.B. Effects of maternal smoking and altitude on birth weight and gestation. In: Reed, D.M. \& Stanley, F.J., eds. The epidemiology of prematurity. Baltimore, Urban \& Schwarzenberg, 1977. p. $81 \cdot 104$.
20. MEYER, M.H. et al. Perinatal events associated with maternal smoking during pregnancy. Amer. J. Epidem., 103:46476. 1976.

21. MONTEIRO, C.A. et al. Estudo da eficácia da suplementação alimentar na gestação no controle do baixo peso ao nascer. [Coletânea de Trabalhos da $1 .^{\mathrm{a}}$ Reunião sobre Metodologia da Investigação Científica em Saúde, Itaparica, BA, 1984, organizada pelo CNPp].

22. QUICK, J.D. et al. Prenatal care and pregnancy outcome in an HMO and general population: a multivariate cohort analysis. Amer. J. publ. H/th, 71:381-90. 1981.

23. SOUZA, J.M.P. de \& BENICIO, M.H.D'A. Análise multivariada: um exemplo usando modelo log-linear. Rev. Saúde públ., S. Paulo, 19:263-9, 1985.

24. WEISS, W. \& JACKSON, E.C. Factores maternos que afetan el peso al nacer. In: Organización Panamericana de la Salud. Factores perinatales que afectan el desarrollo humano. Washington, D.C., 1972. p. 54-8. (OPAS - Publ, cient., 185).

25. WIENER, G. \& MILTON, T. Demografic correlates of low birth weight. Amer. J. Epidem., 91:260-72, 1970.

26. WIENER, G.R. et al. Correlates of low birth-weight: psychological status at eight to ten years of age. Pediat. Res., 2:110. $8,1968$.

27. WORLD HEALTH ORGANIZATION. Division of Family Health. The incidence of low birth weight: a critical review of available information. Wld Hlth Stat. Quart. Rep., 33:197-204, 1980.

Recebido para publicação em 22/01/1985

Aprovado para publicação em 21/05/1985 